\title{
Synthesis of large-scale models: Theory and implementation in an industrial case
}

\author{
J. Pieter Schmal, Johan Grievink, Peter J.T. Verheijen \\ Technical University Delft, Department of Chemical Engineering \\ Julianalaan 136, 2628 BL Delft, The Netherlands
}

\begin{abstract}
Four different model synthesis approaches from different authors are discussed and a variant was developed based on experiences gained with the modelling of a petrochemical plant. A qualitative improvement in model synthesis has been achieved by balancing formalism with practical manageability. Some practical implementation issues are given.
\end{abstract}

\section{Introduction}

Over the years many people have contributed to development of the model building process (e.g. Aris 1994, Murthy et al. 1990, Marquardt 1995, Lohmann and Marquardt 1996, Hangos and Cameron 2001a). The development of models is similar to the design procedure of technical artefacts in general. Hence, the modelling procedure reflects the five steps of the generic design cycle: specifying the functional requirements, assessment of existing domain knowledge, synthesis of model structure, computation and analysis of behaviour, evaluation of model performance based on validation with plant data. The synthesis phase has received little attention in literature, as became apparent when the authors developed a model for a process that was part of a comprehensive cooperation project between industry and academia.

This project, INCOOP (INtegration process unit COntrol and plant-wide OPtimisation), researches the next generation of model based control and dynamic real time optimisation, techniques that rely on rigorous models.

In this article we will discuss our experiences in model synthesis based on an industrial case study. First we will describe the case study, before we discuss the model synthesis theory and compare it with existing approaches. After this we will give guidelines for large-scale model building and discuss implementation issues before we end with conclusions.

\section{Case study}

Within INCOOP a model of a petro-chemical plant has been built (figure 1). The plant contains heat integration, multiple recycles and multiple high purity separations, making it a complex non-linear plant. One single dynamic model was to be the base for the analysis of the plant behaviour for control, and optimisation. Furthermore, the model should function as plant replacement in the test phase of the new techniques. The model had to have high level of detail, sufficient accuracy, flexibility and robustness. The most 
complex version of the DAE model of the plant contains 2.000 differential and 23.000 algebraic equations.

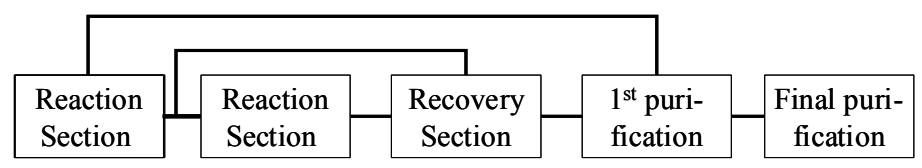

Figure 1: Block diagram of a petro-chemical plant

\section{Model synthesis theory}

We will assume that we have a properly defined modelling goal and we do allow for some iteration in both goal and model structure. Although Aris (1994) states that the formulation is nothing more than rational accounting, especially in the case of largescale models some formalism is needed to minimize model errors. This formalism starts with defining the model building blocks used to set-up the model.

Two formal descriptions of model building blocks can be found in literature. Willems (2000) distinguishes between nodes that correspond with the different blocks; edges with connections between these blocks, terminals with connections between nodes or devices and devices contain laws for change. One level consists of edges, terminals and leafs (a terminal that is not connected). Another level consists of terminals and devices only. A terminal can only be associated with two real variables that reflect a force and the accompanying flux.

Marquardt's (1995) approach is also based on the idea of fluxes and forces. The fluxes are calculated in the connections and the forces in the devices. Marquardt splits the material entities in devices and connections, which in turn consist of composite or elementary elements. In Marquardt's approach a connection may well be a device on a lower level and an explicit distinction is made between (predominantly electrical) signals and phase flows.

Other non-formal building blocks can be found in the method of Lefkopoulos and Stadtherr (1993) that only contains equations and Cameron and Hangos (2001a) that contains different levels of procedures, i.e. procedures and sub-procedures.

The practical implementation led us to a more applicable approach. This approach consists of connections and devices with leaves to indicate one-sided connections. A connection can send multiple variables across the connection, not restricted to force flux combinations. A device may consist of devices coupled by connections, whereas a connection cannot be decomposed.

The model process is to a large extent driven by specific variables that are deduced from the model goal. In our case study the model has wide range of applications from plant replacement to optimisation and thus a wide range of variables needs to be modelled.

\subsection{Abstraction}

The abstraction is concerned with the translation of reality to a model and is thus one of the first steps in the synthesis. Every abstraction calls for a clear definition, place and character, of the system boundary. This character can be closed or open with respect to a phenomenon or variable. Reductions on the other hand are performed within the 
model. Abstractions and reductions are a direct result of the model goal and the capabilities of both modeller and (numerical) solvers. The abstractions and reductions can be of the following types:

- $\quad$ Space (e.g. minimum length scale considered $1 \mathrm{~cm}$ for instance)

- $\quad$ Time (e.g. fastest process considered dynamically $10 \mathrm{~s}$ )

- Phenomena (e.g. no liquid entrainment in distillation)

- $\quad$ Forced (e.g. lack of knowledge)

- $\quad$ Solver based (e.g. ignoring very small numbers)

Aris (1994) pays ample attention to abstractions and reductions, but in the sense of examples rather than an approach. Hangos and Cameron (2001a) point out that it is important to filter the set of all 'model controlling' mechanisms with respect to the modelling goal. In our case study we abstract our plant to figure 1 and define the system boundaries. For simplicity we start with boundaries at the walls of the equipment closed with respect to the surroundings except for explicit in- and outputs. The time scale we are interested in is in the range of seconds to days (time reduction). In a first step we considered two phases on our equilibrium tray and encountered an index problem later on that could not be handled by gPROMS, our modelling environment, forcing us to something different like describing the phases combined (solver based reduction).

\subsection{Decomposition}

Decomposition of the problem reduces the complexity and ensures the problem becomes manageable. The decomposition can be done on two levels as indicated by Marquardt (1994): structural and equational.

\section{Structural decomposition}

The structural decomposition concerns breaking down the original problem in smaller blocks, which contain equations that have something in common depending on how the system was decomposed. For every structural decomposition we need to define an abstraction. The way we decompose the problem can be (for both levels) the following classes:

- Locational: location (time, space or in process stream) is similar

- Tree: natural connection (a direct physical, causal or mathematical connection)

- Behavioural: similar behaviour

- Temporal: similar time scales

- Spatial: similar size

- Functional: similar functions (both operational and mathematical)

Orthogonal to these decompositions are the levels of detail and the levels of hierarchy.

\section{Equational decomposition}

The equational decomposition concerns the break down of equations in a block to sets of equations with a certain similarity. Hangos and Cameron (2001a) give a functional equational decomposition by splitting up the equations in one of the following classes: balance, transfer rate, property relation, balance volume relation, and equipment and control constraint. 


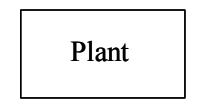

Level: 1

\section{Final puri-
fication}

2

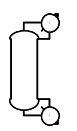

3

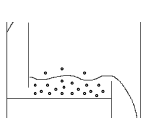

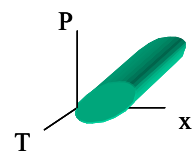

5

Figure 2: Locational and functional structural decomposition

In our case study we break down the plant into sections as in figure 1 and next into devices. A distillation column is further decomposed into trays, a tray into a physical property part and a device specific part. This is a five-level (figure 2) locational structural decomposition with a functional structural decomposition on the last level. In our case we had two different types of physical property relations that could easily be switched due to the functional structural decomposition. A tree equational decomposition was used to decompose the equations (figure 3).

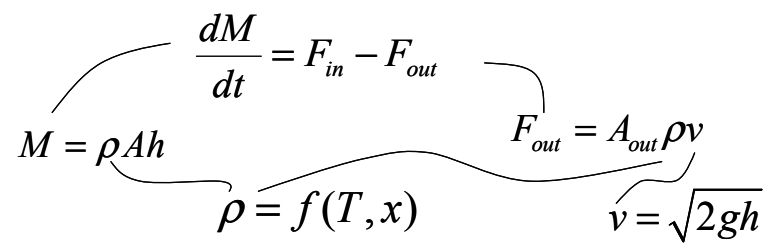

Figure 3: Tree equational aggregation

\subsection{Aggregation}

In contrast with the decomposition we start with equational aggregation since we can only do structural aggregation once the blocks are composed.

\section{Equational aggregation}

The equations in a block must be set-up in such a way that the block can function according to its specifications, i.e. it must fulfil the sub-task it received during the structural decomposition. The equations must be written down in accordance with the way they were decomposed, because it determines the logic needed to minimize errors. The composed blocks are the devices of our model.

\section{Structural aggregation}

The structural aggregation combines the composed blocks, the devices, with the help of connections and creates connections to the surroundings via leafs. The connections describe the information that is shared between devices. Connections and leafs arise from the character of the system boundary of the device that is open.

We choose to use a tree equational decomposition, therefore we start with the component balance (since we were among others interested in product purity). We write down the balance equation first and make sure all variables that occur in the balance are either specified or given by an additional equation (figure 3 for an example of a liquid vessel). Additional assumptions such as equilibrium on the tray could be incorporated at this stage. Next we construct the column by incorporating the other trays, condenser and 
reboiler. To construct the purification section (figure 2) we couple all devices in this section before we hook it to the rest of the plant.

\subsection{Level of detail}

As stated before orthogonal to the decomposition and aggregation above we have the level of detail. Changing the level of detail can be caused by a need for more accuracy or detail, but also by a need for faster simulations or less complexity. Since the systems functions as a whole, the lowest level of detail usually determines the level of detail for the whole system and the highest level of detail usually determines the speed of the simulation, but certainly not always!

In our case study, we increased the level of detail by incorporating the heat-integration and decreased the level of accuracy in the physical properties to increase simulation speed, making the levels of detail more balanced over the system.

\section{Implementation issues}

The way the equations are set-up can have a great influence on the (re-)initialisation and numerical stability. Physically correct alterations due to discontinuities or unwanted limit behaviour are preferred, but may not always be available. In our case study we helped the initialisation by reverting to two directional flows, despite the discontinuities we had to introduce, because the operating region of the model was greatly enlarged.

\section{Discussion}

As stated by Foss et al (1998) the modelling process is poorly understood. This is caused by the fact that we are unable to predict the effect of some choices on the outcome of the simulation. It is the experiences of a modeller with respect to these sensitivities that play an important role during the model synthesis. Some experiences in the sense of pitfalls are given in Murthy et al. (1990).

Table 1: Main differences

\begin{tabular}{lccccc}
\hline & Willems & Marquardt & Hangos & Lefkopoulos & Presented \\
\hline Complementary & Yes & No & Yes & No & Yes \\
Formality & High & High & Low & Low & Medium \\
Number of components & High & Medium & Low & High & Low \\
Strategy levels & 2 & 2 & $>2$ & 1 & 2 \\
\hline
\end{tabular}

The main differences between the leading synthesis methods and our approach are given in table 1. Both Willems and Marquardt have defined a set of rules that should be obeyed for their devices and terminals or connections. In the case of Willems the terminals can only be associated with two real variables that are related in some sense, making the system unnecessary large (number of components). Marquardt's approach, in which a connection may become a device on a lower level, actually embodies multiple models and is therefore not complementary or has some overlap. Hangos' approach on the other hand is too unstructured (low formality) in the sense that there is no systematic way of setting up a consistent set of equations. Our approach is basically 
using the advantages of both Willems, Marquardt and Hangos approach. It balances the need to avoid errors by formality and the manageability to limit model building time.

The modelling tool heavily influences the modelling process (Foss et al. 1998), and thus usually determines the way we decompose our system. Since we used gPROMS in our case study, the model in the examples is well suited for equation-oriented solvers. Especially in the case of large-scale systems causality is usually difficult to determine and parallel equation-oriented solvers are then preferred.

During the aggregation of equations the choice for certain equations is made. It is well known that conservation laws increase numerical stability (Gershenfeld 1999). Whether making the model more linear really helps is questionable, the numerical solver has less problems, but larger inaccuracies in variables occur. For structural aggregation it is important that the streams between the blocks are clearly defined and connecting streams contain the same set of variables. A useful sub-class of the forced reductions are the connection breakers. A connection breaker simply fixes some variables in the connection, e.g. a temperature.

A proper decomposition in combination with modularity can help to change the level of detail from simple (at the start) to complex. The needed level of detail can best be obtained by investigating the levels below and above the current level of detail to see whether a step in either direction is useful.

Finally, model misuse poses a serious thread in large-scale models. Assumptions should therefore be translated into constraints where possible (Hangos and Cameron 2001b).

\section{Conclusions}

Using a model synthesis approach makes the synthesis qualitatively more efficient and robust to errors. The different model syntheses are comparable and the main differences are given in table 1 . The key point of our approach compared to the others is that it balances the formality with practical manageability. Future work consists of investigation of the advantages of different decomposition strategies.

\section{References}

Aris, R., 1994, Mathematical modelling techniques, General Publishing Company Ltd.

Foss, B.A., B. Lohmann, and W. Marquardt, 1998, J.Proc.Cont. 8 (5-6), 325.

Gershenfeld, N., 1999, The nature of mathematical modelling, Cambridge University Press.

Hangos, K., and I. Cameron, 2001a, Process modelling and model analysis, Academic Press.

Hangos, K., and I. Cameron, 2001b, Comp. Chem. Engng. 25, 237

Lefkopoulos, A., and M.A. Stadtherr, 1993, Comp. Chem. Engng. 17, 399.

Lohmann, B., and W. Marquardt, 1996, Comp. Chem. Engng. 20, S213.

Marquardt, W., 1995, Methods of Model-Based Control, NATO-ASI Ser. E, Applied Sciences 293, 3, Kluwer Academic Publ.

Murthy, D.N.P., N.W. Page, and E.Y. Rodin, 1990, Mathematical modelling, Pergamon Press.

Willems, J.C., 2000, Math. Computers in Sim. 53, 227. 